\title{
A
}

SELECCIONES MATEMÁTICAS

Universidad Nacional de Trujillo

ISSN: 2411-1783 (Online)

2020; Vol. 7(2): 267-275.

\section{A note on the conjecture of the autotopism group of the Figueroa's presemifields}

\section{Una nota sobre la conjetura del grupo de autotopismos de los presemicuerpos de Figueroa}

\author{
Walter Meléndez F.(1) and Moisés Delgado O.(i)
}

Received, Oct. 19, 2020

Accepted, Dec. 11, 2020

How to cite this article:

Meléndez FW, Delgado OM. A note on the conjecture of the autotopism group of the Figueroa's presemifields. Selecciones Matemáticas. 2020;7(2):267-275. http://dx.doi.org/10.17268/se1.mat.2020.02.09

\begin{abstract}
In [4] was stated the following conjecture: If a Figueroa's presemifield $P(K, \alpha, \beta, A, B)$ admits an autotopism of order a p-primitive prime divisor of $p^{n}-1$, then its autotopism group is isomorphic to a subgroup of $\Gamma L(K) \times \Gamma L(K)$. In [5] this conjecture was settled under an additional normality condition. In this article, we show that the assumption in the hypothesis of the conjecture is necessary in the sense that there exist a Figueroa's presemifield, that does not admit such autotopism, for which the conjecture is not met.

Keywords . finite presemifield, finite semifield, autotopism group, Cordero-Figueroa semifield, Figueroa's presemifield.
\end{abstract}

\section{Resumen}

En [4] se estableció la siguiente conjetura: Si un presemicuerpo de Figueroa $P(K, \alpha, \beta, A, B)$ admite un autotopismo de orden un divisor primo p-primitivo de $p^{n}-1$, entonces su grupo autotopismo es isomórfo a un subgrupo de $\Gamma L(K) \times \Gamma L(K)$. En [5] esta conjetura se resolvió bajo una condición adicional de normalidad. En este artículo, mostramos que la suposición hecha en la hipótesis de la conjetura es necesaria en el sentido de que existe un presemicuerpo de Figueroa, que no admite tal autotopismo, para el cual la conjetura no se cumple.

Palabras clave. presemicuerpo finito, semicuerpo finito, grupo autotopismo, semicuerpo de Cordero-Figueroa, presemicuerpo de Figueroa.

\section{Introduction.}

Definition 1.1. A finite presemifield $(P,+, *)$ consists of an additive group $(P,+)$ and a multiplication * that satisfies both distributive laws and the condition: if $x * y=0$ then $x=0$ or $y=0$. A finite presemifield with multiplicative identity is called finite semifield.

Throughout this article, the term presemifield (or semifield) will always be used to refer a finite presemifield (or a finite semifield).

Definition 1.2. Two presemifields (or semifields) $(P,+, *)$ and $\left(P^{\prime},+, \circ\right)$ are isotopic if there exist a triple $(F, G, H)$ of bijective functions from $P$ to $P^{\prime}$ which are additives and satisfy $G(x * y)=F(x) \circ H(y)$, for all $x, y \in P$. The triple $(F, G, H)$ is called an isotopism from $P$ to $P^{\prime}$.

An isotopism from a presemifield (or semifield) $P$ to itself is called an autotopism of $P$, and the set of all autotopisms of a presemifield (or semifield) $P$ is known as the autotopism group of $P$ and will be denoted by $\mathcal{A}(P)$. For more details of our concern about semifields, presemifields, autotopisms, and the autotopism group $\mathcal{A}(P)$, see [4] and [5].

\footnotetext{
*Facultad de Ciencias Físicas y Matemáticas, Universidad Nacional de Trujillo, Avenida Juan Pablo II s/n, Trujillo, Perú (wmelendezeunitru.edu.pe).

${ }^{\dagger}$ Mathematics and Physics Department, University of Puerto Rico, Cayey Campus, 205 Calle Antonio R. Barceló, Cayey, Puerto Rico (moises. delgado@upr.edu).
} 
In this paper, we will focus our attention on the conjecture stated in [5] about the autotopism group of the Figueroa's presemifield of order $p^{n}$. A Figueroa's presemifield of order $p^{n}$ is defined in [4] as follows:

Definition 1.3. Let $\alpha \neq 1$ and $\beta \neq 1, \alpha \neq \beta$, be automorphisms of $K=G F\left(p^{n}\right)$, where $p \geqslant 3$ and $n \geqslant 4$, and let $A, B \in K^{*}$ be constants. $(K,+, *)$ is a Figueroa's presemifield of order $p^{n}$ with the product:

$$
x * y=x y+A x^{\alpha} y^{\beta}+B x^{\beta} y^{\alpha},
$$

if $\alpha, \beta, A$, and $B$ are such that $x * y=0$ implies $x=0$ or $y=0$. This presemifield is denoted by $P(K, \alpha, \beta, A, B)$.

In [4] we studied the autotopism group of the Cordero-Figueroa semifield of order $3^{6}$ and we showed that its autotopism group is isomorphic to a particular subgroup of $\Gamma L(K) \times \Gamma L(K)$. There in, because of additional evidence, we also suggested that the same fact should be fulfilled for the general case (i.e. for a Figueroa's presemifield of order $p^{n}$ ). Thus, in [5], we formally conjecture:

Conjecture 1 (Figueroa's conjecture). If a Figueroa's presemifield $P(K, \alpha, \beta, A, B)$ of order $p^{n}$ admits an autotopism of order a p-primitive prime divisor, then its autotopism group is isomorphic to a subgroup of $\Gamma L(K) \times \Gamma L(K)$.

In [5], this conjecture was proved as true under the additional condition that the subgroup generated by the autotopism $\left(f_{0} x, f_{0}^{2} y, f_{0} n\right) \in \mathcal{A}(P)$, of an apropriate order, be a normal subgroup in $\mathcal{A}(P)$.

The goal in this article is to show that the hypothesis of the Figueroa's conjecture is necessary in the sense that there exist an example where the hypothesis is not satisfied and the conjecture for this example is not met.

2. Resolved cases. In this section, we review the main results obtained in [4] and [5] that contribute to the proof of the stated conjecture.

2.1. The autotopism group of the Cordero-Figueroa semifield of order $3^{6}$. In [4], we determine that the full autotopism group of the Cordero-Figueroa semifield of order $3^{6}$ is isomorphic to a particular subgroup of $\Gamma L(K) \times \Gamma L(K)$, where $K=G F\left(3^{6}\right)$. To be more specific:

Theorem 2.1. Let $P=P(K, \alpha, \beta, A, B)$ be the Cordero-Figueroa semifield of order $3^{6}$. The full autotopism group $\mathcal{A}(P)$ is isomorphic to the subgroup of $\Gamma L(K) \times \Gamma L(K)$ :

$$
\left\langle\left(\gamma^{13} x, \gamma^{26} y\right)\right\rangle \rtimes\left\langle\left(\gamma x^{3}, \gamma y^{3}\right)\right\rangle
$$

where $\left\langle\left(\gamma^{13} x, \gamma^{26} y\right)\right\rangle$ is normal in the group $\left\langle\left(\gamma^{13} x, \gamma^{26} y\right)\right\rangle\left\langle\left(\gamma x^{3}, \gamma y^{3}\right)\right\rangle$. Furthermore, the order of $\mathcal{A}(P)$ is 672 .

Since the Cordero-Figueroa semifield of order $3^{6}$ is a semifield that admits an autotopism of order a 3primitive prime divisor of $3^{6}-1$, it is a presemifield (see [1]), or more properly speaking, it is a Figueroa's presemifield of order $3^{6}$.

2.2. The autotopism group of the Figueroa's presemifield of order $p^{n}$. In [5], in order to prove the Figueroa's conjecture, we provided a characterization for a Figueroa's presemifield of order $p^{n}$ to admit a certain autotopism of order a $p$-primitive prime divisor of $p^{n}-1$, as follows:

Theorem 2.2. Let $P=P(K, \alpha, \beta, A, B)$ be a Figueroa's presemifield of order $p^{n}$. Assume that $p^{n}-1$ has a p-primitive prime divisor $s$ and $\alpha^{3} \neq 1$ if $\alpha \beta=1$. Then $\mathcal{A}(P)$ admits the autotopism $\left(f_{0} x, f_{0}^{2} y, f_{0} n\right)$ of order $s$, with $f_{0} \in K^{*}$, if and only if $s$ divides $(\beta-1)+(\alpha-1)$.

This characterization allowed us to demonstrate the Figueroa's conjecture in Theorem 2.3, under the assumption that the subgroup generated by the autotopism $\left(f_{0} x, f_{0}^{2} y, f_{0} n\right) \in \mathcal{A}(P)$ is a normal subgroup in $\mathcal{A}(P)$.

Theorem 2.3. Let $P=P(K, \alpha, \beta, A, B)$ be a Figueroa's presemifield of order $p^{n}$. Assume that $\mathcal{A}(P)$ admits the autotopism of order $s$ (as referred in Theorem 2.2). If the subgroup generated by this autotopism is normal in $\mathcal{A}(P)$, then

$$
\mathcal{A}(P)=\left\{\left(u x^{\phi}, u v y^{\phi}, v n^{\phi}\right): u, v \in K^{*}, \phi \in A u t(K)\right\},
$$

and hence, it is isomorphic to a subgroup of $\Gamma L(K) \times \Gamma L(K)$.

3. On the sufficient condition of the Figueroa's conjecture. As mentioned at the end of section 1, in this section we provide a Figueroa presemifield of order $3^{4}$ and find its autotopism group. We show that the assumption on the existence of an autotopism of order a $p$-primitive prime divisor of $p^{n}-1$, in the hypothesis of Figueroa's conjecture, is required.

Theorem 3.1. Let $K=G F\left(3^{4}\right)$ and consider $\alpha=3^{1}, \beta=3^{3}, A=\gamma^{0}=1$ and $B=\gamma^{13}$, with $\gamma \in K$ a primitive element such that $\gamma^{4}=1+\gamma$. Let $P=P(K, \alpha, \beta, A, B)$ be the Figueroa's presemifield defined by the product 


$$
x \circ y=x y+A x^{3^{1}} y^{3^{3}}+B x^{3^{3}} y^{3^{1}},
$$

for all $x, y \in K$. Then, $P$ does not admit an autotopism of order a 3-primitive prime divisor of $3^{4}-1$ and $\mathcal{A}(P)$ is not isomorphic to any subgroup of $\Gamma L(K) \times \Gamma L(K)$.

Proof: Let $(f, g, h) \in \mathcal{A}(P)$ be any element. Since $f, g$ and $h$ are additive functions,

$$
f(x)=\sum_{k=0}^{3} f_{k} x^{3^{k}}, g(y)=\sum_{k=0}^{3} g_{k} y^{3^{k}}, h(n)=\sum_{k=0}^{3} h_{k} n^{3^{k}} .
$$

Let $m=h(n)$. Then, the condition $g(x \circ n)=f(x) \circ m$ yields the following equations:

$$
\begin{aligned}
g_{0} n+\left(g_{3}+g_{1} B^{3}\right) n^{3^{2}} & =f_{0} m+f_{3}^{3^{1}} m^{3^{3}}+B f_{1}^{3^{3}} m^{3^{1}}, \\
g_{1} n^{3^{1}}+\left(g_{0}+g_{2} B\right) n^{3^{3}} & =f_{1} m+f_{0}^{3^{1}} m^{3^{3}}+B f_{2}^{3^{3}} m^{3^{1}} \\
\left(g_{1}+g_{3} B^{3}\right) n+g_{2} n^{3^{2}} & =f_{2} m+f_{1}^{3^{1}} m^{3^{3}}+B f_{3}^{3^{3}} m^{3^{1}} \\
\left(g_{2}+g_{0} B\right) n^{3^{1}}+g_{3} n^{3^{3}} & =f_{3} m+f_{2}^{3^{1}} m^{3^{3}}+B f_{0}^{3^{3}} m^{3^{1}} .
\end{aligned}
$$

Since the multiplicative group of the middle nucleus of $P$ :

$$
N_{m}^{*}=\left\{(f, g, h) \in \mathcal{A}(P): f(x)=c x, g(y)=y, h(n)=c^{-1} n\right\}, .
$$

is normal in $\mathcal{A}(P)$ (see [4]), we have that for all $(f, g, h) \in \mathcal{A}(P)$ and any $\left(f_{0}, i, h_{0}\right) \in N_{m}^{*}$, where $i$ is the identity function from $P$ to $P$, there exists $\left(\tilde{f}_{0}, i, \tilde{h}_{0}\right) \in N_{m}^{*}$ such that

$$
(f, g, h)^{-1} \circ\left(f_{0}, i, h_{0}\right) \circ(f, g, h)=\left(\tilde{f}_{0}, i, \tilde{h}_{0}\right) .
$$

Then, for all $x \in K$,

$$
f_{0}(f(x))=f\left(\tilde{f}_{0}(x)\right) .
$$

Let $f_{0}(x)=c_{0} x$ and $\tilde{f}_{0}(x)=\tilde{c}_{0} x$. Since $\left|N_{m}^{*}\right|=3^{(4,3-1)}-1=8$, we get that $\tilde{c}_{0} \in G F^{*}\left(3^{2}\right)$ (see Lemma 1 in [4]). Then, from (3.5),

$$
\begin{aligned}
& c_{0} f_{0}=f_{0} \tilde{c}_{0}, \\
& c_{0} f_{1}=f_{1} \tilde{c}_{0}^{3}, \\
& c_{0} f_{2}=f_{2} \tilde{c}_{0}, \\
& c_{0} f_{3}=f_{3} \tilde{c}_{0}^{3} .
\end{aligned}
$$

Since $f(x) \neq 0, f(x)$ has at least one nonzero coefficient. Suppose that $f_{0} \neq 0$. Then, from (3.6), $c_{0}=\tilde{c}_{0}$. Hence, (3.7) and (3.9) imply that $f_{1}=f_{3}=0$. In the same way, if $f_{2} \neq 0$ then $f_{1}=f_{3}=0$, and if $f_{1} \neq 0$ or $f_{3} \neq 0$ then $f_{0}=f_{2}=0$. Therefore, $f(x)$ has at least one nonzero coefficient and at most two nonzero coefficients $\left(f_{0}\right.$ and $f_{2}$, or $f_{1}$ and $\left.f_{3}\right)$. We now analyze each case in order to determine the full autotopism group of $P$.

CASE 1: Two coefficients of $f(x)$ are nonzero. For this case, we have the following subcases:

(a) $f_{0} \neq 0, f_{2} \neq 0, f_{1}=0, f_{3}=0$.

(b) $f_{1} \neq 0, f_{3} \neq 0, f_{2}=0, f_{4}=0$.

If (a) occurs, then (3.1) - (3.4) imply that

$$
\begin{aligned}
m & =\frac{g_{0}}{f_{0}} n+\left(\frac{g_{3}}{f_{0}}+\frac{g_{1}}{f_{0}} B^{3}\right) n^{9}, \\
f_{0}^{9} m+B^{3} f_{2} m^{9} & =\left(g_{0}^{3}+g_{2}^{3} B^{3}\right) n+g_{1}^{3} n^{9}, \\
m & =\left(\frac{g_{1}}{f_{2}}+\frac{g_{3}}{f_{2}} B^{3}\right) n+\frac{g_{2}}{f_{2}} n^{9}, \\
f_{2}^{9} m+B^{3} f_{0} m^{9} & =g_{3}^{3} n+\left(g_{2}^{3}+g_{0}^{3} B^{3}\right) n^{9} .
\end{aligned}
$$

From (3.10) and (3.12), we get

$$
\begin{aligned}
& \frac{g_{0}}{f_{0}}=\frac{g_{1}}{f_{2}}+\frac{g_{3}}{f_{2}} B^{3}, \\
& \frac{g_{2}}{f_{2}}=\frac{g_{3}}{f_{0}}+\frac{g_{1}}{f_{0}} B^{3} .
\end{aligned}
$$


Let $P=\frac{g_{2}}{f_{2}}$ and $Q=\frac{g_{0}}{f_{0}}$. Then, from equations (3.14) and (3.15) we obtain

$$
\begin{aligned}
& f_{2} Q=g_{1}+g_{3} B^{3}, \\
& f_{0} P=g_{3}+g_{1} B^{3} .
\end{aligned}
$$

Hence

$$
\begin{aligned}
& g_{1}=-\left(f_{0} P+f_{2} Q B\right), \\
& g_{3}=-\left(f_{2} Q+f_{0} P B\right) .
\end{aligned}
$$

On the other hand, from (3.10) and (3.15) we conclude that

$$
m=\frac{g_{0}}{f_{0}} n+\frac{g_{2}}{f_{2}} n^{3^{2}},
$$

or

$$
m=Q n+P n^{9} .
$$

Substituting this into equation (3.11) we obtain

$$
f_{0}^{9}\left(Q n+P n^{9}\right)+B^{3} f_{2}\left(Q^{9} n^{9}+P^{9} n\right)=\left(g_{0}^{3}+g_{2}^{3} B^{3}\right) n+g_{1}^{3} n^{9},
$$

which is equivalent to

$$
\left(f_{0}^{9} Q+B^{3} f_{2} P^{9}\right) n+\left(f_{0}^{9} P+B^{3} f_{2} Q^{9}\right) n^{9}=\left(g_{0}^{3}+g_{2}^{3} B^{3}\right) n+g_{1}^{3} n^{9},
$$

a polynomial identity in $n$. Therefore

$$
\begin{aligned}
& f_{0}^{9} Q+B^{3} f_{2} P^{9}=g_{0}^{3}+g_{2}^{3} B^{3}, \\
& f_{0}^{9} P+B^{3} f_{2} Q^{9}=g_{1}^{3} .
\end{aligned}
$$

Since $g_{0}=f_{0} Q$ and $g_{2}=f_{2} P$, the equations (3.19), (3.20) and (3.16) imply that

$$
\begin{aligned}
& f_{0}^{9} Q+B^{3} f_{2} P^{9}=f_{0}^{3} Q^{3}+f_{2}^{3} P^{3} B^{3}, \\
& f_{0}^{9} P+B^{3} f_{2} Q^{9}=-\left(f_{0}^{3} P^{3}+f_{2}^{3} Q^{3} B^{3}\right) .
\end{aligned}
$$

Notice that $B^{4}=-1$. Then, multiplying (3.21) and (3.22) by $B$, and rearranging the resultant equations, we get

$$
\begin{aligned}
& \left(f_{0}^{9} Q-f_{0}^{3} Q^{3}\right) B=f_{2} P^{9}-f_{2}^{3} P^{3}, \\
& \left(f_{0}^{9} P+f_{0}^{3} P^{3}\right) B=f_{2} Q^{9}+f_{2}^{3} Q^{3} .
\end{aligned}
$$

Similarly, replacing the expression obtained for $m$ (see equation (3.18)) into equation (3.13), we obtain that

$$
\begin{aligned}
& \left(f_{2}^{9} Q+f_{2}^{3} Q^{3}\right) B=f_{0} P^{9}+f_{0}^{3} P^{3}, \\
& \left(f_{2}^{9} P-f_{2}^{3} P^{3}\right) B=f_{0} Q^{9}-f_{0}^{3} Q^{3} .
\end{aligned}
$$

Now, adding and subtracting side to side (3.23) and (3.24) we find, respectively, that

$$
\begin{aligned}
& f_{0}^{9} B(P+Q)+f_{0}^{3} B(P-Q)^{3}=f_{2}(P+Q)^{9}+f_{2}^{3}(Q-P)^{3}, \\
& f_{0}^{9} B(P-Q)+f_{0}^{3} B(P+Q)^{3}=f_{2}(Q-P)^{9}+f_{2}^{3}(P+Q)^{3} .
\end{aligned}
$$

Analogously, from (3.25) and (3.26), we get

$$
\begin{aligned}
& f_{2}^{9} B(P+Q)+f_{2}^{3} B(Q-P)^{3}=f_{0}(P+Q)^{9}+f_{0}^{3}(P-Q)^{3}, \\
& f_{2}^{9} B(Q-P)+f_{2}^{3} B(P+Q)^{3}=f_{0}(P-Q)^{9}+f_{0}^{3}(P+Q)^{3} .
\end{aligned}
$$

Let $R=P+Q$ and $S=P-Q$. Then, from (3.27) - (3.30),

$$
\begin{aligned}
f_{0}^{9} B R+f_{0}^{3} B S^{3} & =f_{2} R^{9}-f_{2}^{3} S^{3}, \\
f_{0}^{9} B S+f_{0}^{3} B R^{3} & =-f_{2} S^{9}+f_{2}^{3} R^{3}, \\
f_{2}^{9} B R-f_{2}^{3} B S^{3} & =f_{0} R^{9}+f_{0}^{3} S^{3}, \\
-f_{2}^{9} B S+f_{2}^{3} B R^{3} & =f_{0} S^{9}+f_{0}^{3} R^{3} .
\end{aligned}
$$


Rearranging terms in each one of these equations, we obtain

$$
\begin{aligned}
& \left(f_{2}^{3}+f_{0}^{3} B\right) S^{3}=f_{2} R^{9}-f_{0}^{9} B R, \\
& \left(f_{2}^{3}-f_{0}^{3} B\right) R^{3}=f_{2} S^{9}+f_{0}^{9} B S, \\
& \left(f_{0}^{3}+f_{2}^{3} B\right) S^{3}=f_{2}^{9} B R-f_{0} R^{9}, \\
& \left(f_{2}^{3} B-f_{0}^{3}\right) R^{3}=f_{0} S^{9}+f_{2}^{9} B S .
\end{aligned}
$$

Multiplying (3.31) by $f_{0}$ and (3.33) by $f_{2}$, and adding side to side the resulting equations, we get

$$
\left[\left(f_{2}^{3}+f_{0}^{3} B\right) f_{0}+\left(f_{0}^{3}+f_{2}^{3} B\right) f_{2}\right] S^{3}=\left(f_{2}^{10}-f_{0}^{10}\right) B R .
$$

In the same way, multiplying (3.31) by $f_{2}^{9}$ and (3.33) by $f_{0}^{9}$, and adding side to side the resulting equations, we obtain that

$$
\left[\left(f_{2}^{3}+f_{0}^{3} B\right) f_{2}^{9}+\left(f_{0}^{3}+f_{2}^{3} B\right) f_{0}^{9}\right] S^{3}=\left(f_{2}^{10}-f_{0}^{10}\right) R^{9} .
$$

Similarly, with (3.32) and (3.34), we have

$$
\begin{aligned}
{\left[\left(f_{2}^{3}-f_{0}^{3} B\right) f_{0}-\left(f_{2}^{3} B-f_{0}^{3}\right) f_{2}\right] R^{3} } & =\left(f_{0}^{10}-f_{2}^{10}\right) B S, \\
{\left[\left(f_{2}^{3}-f_{0}^{3} B\right) f_{2}^{9}-\left(f_{2}^{3} B-f_{0}^{3}\right) f_{0}^{9}\right] R^{3} } & =\left(f_{2}^{10}-f_{0}^{10}\right) S^{9} .
\end{aligned}
$$

Rearranging terms in (3.35) - (3.38), we obtain

$$
\begin{aligned}
{\left[f_{0} f_{2}\left(f_{2}^{2}+f_{0}^{2}\right)+\left(f_{0}^{4}+f_{2}^{4}\right) B\right] S^{3} } & =\left(f_{2}^{10}-f_{0}^{10}\right) B R, \\
{\left[\left(f_{2}^{4}+f_{0}^{4}\right)^{3}+f_{0}^{3} f_{2}^{3}\left(f_{2}^{6}+f_{0}^{6}\right) B\right] S^{3} } & =\left(f_{2}^{10}-f_{0}^{10}\right) R^{9}, \\
{\left[\left(f_{2}^{4}+f_{0}^{4}\right) B-f_{0} f_{2}\left(f_{2}^{2}+f_{0}^{2}\right)\right] R^{3} } & =\left(f_{2}^{10}-f_{0}^{10}\right) B S, \\
{\left[\left(f_{2}^{4}+f_{0}^{4}\right)^{3}-f_{0}^{3} f_{2}^{3}\left(f_{2}^{6}+f_{0}^{6}\right) B\right] R^{3} } & =\left(f_{2}^{10}-f_{0}^{10}\right) S^{9} .
\end{aligned}
$$

Let $V=f_{0} f_{2}\left(f_{2}^{2}+f_{0}^{2}\right), W=f_{0}^{4}+f_{2}^{4}$ and $D=f_{2}^{10}-f_{0}^{10}$. We note that, since $f(x)$ is bijective, $f_{0}^{10} \neq f_{2}^{10}$. Hence, $D \neq 0$. Furthermore, since $D^{9}=f_{2}^{90}-f_{0}^{90}=f_{2}^{10}-f_{0}^{10}=D$, we get $D^{8}=1$. Replacing the expressions $V, W$ and $D$ in (3.39) - (3.42), we obtain

$$
\begin{aligned}
(V+W B) S^{3} & =D B R \\
\left(W^{3}+V^{3} B\right) S^{3} & =D R^{9} \\
(W B-V) R^{3} & =D B S \\
\left(W^{3}-V^{3} B\right) R^{3} & =D S^{9} .
\end{aligned}
$$

From (3.43), if $S=0$ then $R=0$. Since $R=P+Q$ and $S=P-Q$, we conclude that $P=0$ and $Q=0$. Therefore, equation (3.18) implies that $m=0$, which is a contradiction. Analogously, from (3.45), if $R=0$ then $S=0$. Therefore, $m=0$. Hence $R \neq 0$ and $S \neq 0$.

Solving (3.43) for $R$ and replacing it in (3.44) - (3.46), we obtain the following equations

$$
\begin{aligned}
B\left(W^{3}+V^{3} B\right) & =\left(V^{9}+W^{9} B\right) S^{24}, \\
(W B-V)\left(V^{3}+W^{3} B^{3}\right) S^{8} & =-D^{4}, \\
\left(W^{3}-V^{3} B\right)\left(V^{3}+W^{3} B^{3}\right) & =D^{4} B^{3} .
\end{aligned}
$$

Multiplying the equation (3.49) by $B$, we get

$$
\left(W^{3}-V^{3} B\right)^{2}=D^{4} .
$$

Hence

$$
W^{3}-V^{3} B=\epsilon D^{2}, \quad(\epsilon= \pm 1) .
$$

On the other hand, multiplying the equation (3.48) by $B$ and using (3.51), we get

$$
(W B-V) \epsilon S^{8}=D^{2} B .
$$

Hence

$$
\left(W^{3} B^{3}-V^{3}\right) \epsilon S^{24}=D^{6} B^{3} .
$$


Multiplying this equation by $B$, we get

$$
\left(W^{3}+V^{3} B\right) \epsilon S^{24}=D^{6} .
$$

Solving the equation (3.52) for $S^{24}$ and replacing into equation (3.47), we get

$$
B\left(W^{3}+V^{3} B\right)^{2}=\epsilon\left(V^{9}+W^{9} B\right) D^{6} .
$$

Multiplying each side of this equation by $B^{3}$, we get

$$
-\left(W^{3}+V^{3} B\right)^{2}=\epsilon\left(V^{3} B-W^{3}\right)^{3} D^{6} .
$$

From (3.51) and (3.53)

$$
\left(W^{3}+V^{3} B\right)^{2}=D^{4}
$$

Thus (3.50) and (3.54) imply that

$$
\left(W^{3}+V^{3} B\right)^{2}=\left(W^{3}-V^{3} B\right)^{2} .
$$

Then $W=0$ or $V=0$. Suppose that $W=0$. Then (3.54) imply that $V^{6} B^{2}=D^{4}$. So,

$$
V^{12} B^{4}=D^{8} \text {. }
$$

Since $B^{4}=-1$ and $D^{8}=1$, from (3.55), we have that $V^{12}=-1$, which implies that $V^{4}=-1$.

Remember that $W=f_{0}^{4}+f_{2}^{4}$ and $V=f_{0} f_{2}\left(f_{2}^{2}+f_{0}^{2}\right)$. Since $W=0$, we have that $f_{2}^{4}+f_{0}^{4}=0$. From where, $f_{2}^{4}=-f_{0}^{4}$. Thus

$$
V^{2}=f_{0}^{2} f_{2}^{2}\left(f_{2}^{2}+f_{0}^{2}\right)^{2}=2 f_{0}^{4} f_{2}^{4}=-f_{0}^{4}\left(-f_{0}^{4}\right)=f_{0}^{8} .
$$

Then

$$
f_{0}^{16}=V^{4}=-1
$$

Note that (3.56) implies that $f_{0}^{32}=1$. Then $f_{0}^{96}=1$. Since $f_{0}^{80}=1$, we get

$$
f_{0}^{16}=1
$$

which contradicts (3.56). Hence $W \neq 0$ and $V=0$. Since $V=0,(3.50)$ implies

$$
W^{6}=D^{4} \text {. }
$$

Then, $W^{12}=D^{8}=1$. So, $W^{4}=1$. Thus (3.57) implies $W^{2}=D^{4}$. Therefore

$$
W=\tilde{\epsilon} D^{2}, \quad(\tilde{\epsilon}= \pm 1) .
$$

Now, equations (3.51) and (3.58) imply that $W^{3}=W$ or $W^{3}=-W$. Thus, since $W \neq 0$, we conclude that $W^{2}=1$ or $W^{2}=-1$.

Assume first that $W^{2}=1$. Since $V=0$,

$$
f_{0}^{2}+f_{2}^{2}=0 \text {. }
$$

Then $f_{2}^{4}=f_{0}^{4}$. Therefore

$$
W=f_{0}^{4}+f_{2}^{4}=2 f_{0}^{4}=-f_{0}^{4} .
$$

Hence, $W^{2}=f_{0}^{8}$. Thus

$$
f_{0}^{8}=1
$$

Solving the equation (3.59) for $f_{2}$ give us

$$
f_{2}=j f_{0},
$$


where $j \in K^{*}$ is such that $j^{2}=-1$ (then $j= \pm B^{2}$ ). Replacing $f_{2}$ in (3.23) - (3.26), we get

$$
\begin{aligned}
\left(f_{0}^{9} Q-f_{0}^{3} Q^{3}\right) B & =j f_{0} P^{9}+j f_{0}^{3} P^{3}, \\
\left(f_{0}^{9} P+f_{0}^{3} P^{3}\right) B & =j f_{0} Q^{9}-j f_{0}^{3} Q^{3}, \\
\left(j f_{0}^{9} Q-j f_{0}^{3} Q^{3}\right) B & =f_{0} P^{9}+f_{0}^{3} P^{3}, \\
\left(j f_{0}^{9} P+j f_{0}^{3} P^{3}\right) B & =f_{0} Q^{9}-f_{0}^{3} Q^{3} .
\end{aligned}
$$

Multiplying the equation (3.63) by $j$, and using (3.65), we get

$$
j f_{0}^{9} P+j f_{0}^{3} P^{3}=-\left(j f_{0}^{9} P+j f_{0}^{3} P^{3}\right) .
$$

Then

$$
P^{2}=-f_{0}^{6}
$$

Then, since $f_{0}^{8}=1$, from (3.66), we get that $P^{6}=-f_{0}^{18}=-f_{0}^{2}$. Therefore,

$$
f_{0} P^{9}+f_{0}^{3} P^{3}=f_{0} P^{6} P^{3}+f_{0}^{3} P^{3}=f_{0}\left(-f_{0}^{2}\right) P^{3}+f_{0}^{3} P^{3}=-f_{0}^{3} P^{3}+f_{0}^{3} P^{3}=0 .
$$

Thus, the right hand side of (3.64) is 0 . Then, since $B \neq 0$, from (3.64), we get that $j f_{0}^{9} Q-j f_{0}^{3} Q^{3}=0$. Hence

$$
Q^{2}=f_{0}^{6}
$$

Since $f_{2}=j f_{0}$, we get $P=\frac{g_{2}}{f_{2}}=\frac{g_{2}}{j f_{0}}$. Replacing $f_{2}=j f_{0}, P=\frac{g_{2}}{j f_{0}}$ and $Q=\frac{g_{0}}{f_{0}}$ in (3.16) and (3.17), we have

$$
\begin{aligned}
& -g_{1}=\frac{g_{2}}{j}+j B g_{0}, \\
& -g_{3}=j g_{0}+B \frac{g_{2}}{j} .
\end{aligned}
$$

Next, let us find the form of the autotopisms $(f, g, h)$ of the presemifield $P$ for this case:

FORM 1. Solving (3.60) for $f_{0}$ we obtain $f_{0}=B^{k}$, for $k \in \mathbb{N}, 0 \leq k \leq 7$. Since $g_{0}=f_{0} Q$, we get $g_{0}^{2}=f_{0}^{2} Q^{2}$. Then, using (3.67), we find $g_{0}^{2}=f_{0}^{2} f_{0}^{6}=f_{0}^{8}=1$. Therefore, $g_{0}= \pm 1$.

Similarly, since $g_{2}=f_{2} P$, we get that $g_{2}^{2}=f_{2}^{2} P^{2}$. Then, since $f_{2}=j f_{0}$ (where $j^{2}=-1$ ), using (3.66), we find that $g_{2}^{2}=j^{2} f_{0}^{2}\left(-f_{0}^{6}\right)=f_{0}^{8}=1$. Therefore, $g_{2}= \pm 1$.

(i) Assume that $g_{0}=1$ and $g_{2}=1$. If $j=B^{2}$, then from equation (3.68),

$$
g_{1}=-\left(\frac{1}{B^{2}}+B^{3}\right)=B^{2}-B^{3}=-1 .
$$

Similarly, from equation (3.69),

$$
g_{3}=-\left(B^{2}+B \frac{1}{B^{2}}\right)=-B^{2}+B^{3}=1 .
$$

So, we get autotopisms $(f, g, h)$ where

$$
\begin{aligned}
& f(x)=B^{k} x+B^{k+2} x^{9}, \\
& g(y)=y-y^{3}+y^{9}+y^{27}, \\
& h(n)=B^{-k} n+B^{-k-2} n^{9},
\end{aligned}
$$

for $0 \leq k \leq 7$.

In the same way, if $j=-B^{2}$ then $g_{1}=1$ and $g_{3}=-1$. So, we get autotopisms $(f, g, h)$ where

$$
\begin{aligned}
& f(x)=B^{k} x-B^{k+2} x^{9}, \\
& g(y)=y+y^{3}+y^{9}-y^{27}, \\
& h(n)=B^{-k} n-B^{-k-2} n^{9},
\end{aligned}
$$

for $0 \leq k \leq 7$. 
(ii) If $g_{0}=1$ and $g_{2}=-1$, in a similar way to (i), we get autotopisms $(f, g, h)$ where, for $0 \leq k \leq 7$,

$$
\begin{aligned}
& f(x)=B^{k} x+B^{k+2} x^{9} \\
& f(x)=B^{k} x-B^{k+2} x^{9}, \\
& g(y)=y+y^{3}-y^{9}-y^{27} \\
& \text { and } \\
& g(y)=y-y^{3}-y^{9}-y^{27}, \\
& h(n)=B^{-k} n-B^{-k-2} n^{9} \\
& h(n)=B^{-k} n+B^{-k-2} n^{9} .
\end{aligned}
$$

(iii) If $g_{0}=-1$ and $g_{2}=1$, we get autotopisms $(f, g, h)$ where, for $0 \leq k \leq 7$,

$$
\begin{aligned}
& f(x)=B^{k} x+B^{k+2} x^{9} \\
& g(y)=-y-y^{3}+y^{9}-y^{27} \\
& \text { and } \\
& f(x)=B^{k} x-B^{k+2} x^{9}, \\
& h(n)=-B^{-k} n+B^{-k-2} n^{9} \\
& g(y)=-y-y^{3}+y^{9}+y^{27}, \\
& h(n)=-B^{-k} n-B^{-k-2} n^{9} \text {. }
\end{aligned}
$$

(iv) If $g_{0}=-1$ and $g_{2}=-1$, we get autotopisms $(f, g, h)$ where, for $0 \leq k \leq 7$,

$$
\begin{aligned}
& f(x)=B^{k} x+B^{k+2} x^{9} \\
& f(x)=B^{k} x-B^{k+2} x^{9}, \\
& g(y)=-y+y^{3}-y^{9}-y^{27} \\
& \text { and } \\
& g(y)=-y-y^{3}-y^{9}+y^{27}, \\
& h(n)=-B^{-k} n-B^{-k-2} n^{9} \\
& h(n)=-B^{-k} n+B^{-k-2} n^{9} \text {. }
\end{aligned}
$$

FORM 2. Assume that $W^{2}=-1$. As when $W^{2}=1$ (see page 272), we get autotopisms $(f, g, h)$ where, for $0 \leq k \leq 7$,

(i)

(ii)

$$
\begin{aligned}
& f(x)=\gamma^{5} B^{k} x+\gamma^{5} B^{k+2} x^{9} \\
& g(y)=B^{2} y-B^{2} y^{3}+B^{2} y^{9}+B^{2} y^{27} \quad \text { and } \\
& f(x)=\gamma^{5} B^{k} x-\gamma^{5} B^{k+2} x^{9}, \\
& h(n)=\gamma^{-5} B^{2-k} n+\gamma^{-5} B^{-k} n^{9} \\
& g(y)=B^{2} y+B^{2} y^{3}+B^{2} y^{9}-B^{2} y^{27} \text {, } \\
& h(n)=\gamma^{-5} B^{2-k} n-\gamma^{-5} B^{-k} n^{9} \text {. }
\end{aligned}
$$

$$
\begin{aligned}
f(x) & =\gamma^{5} B^{k} x+\gamma^{5} B^{k+2} x^{9} \\
g(y) & =B^{2} y-B^{3} y^{3}-B^{2} y^{9}-B^{3} y^{27} \quad \text { and } \\
h(n) & =\gamma^{-5} B^{2-k} n-\gamma^{-5} B^{-k} n^{9}
\end{aligned}
$$$$
f(x)=\gamma^{5} B^{k} x-\gamma^{5} B^{k+2} x^{9},
$$

(iii)

$$
\begin{aligned}
& f(x)=\gamma^{5} B^{k} x+\gamma^{5} B^{k+2} x^{9} \\
& g(y)=-B^{2} y+B^{3} y^{3}-+B^{2} y^{9}+B^{3} y^{27} \quad \text { and } \\
& f(x)=\gamma^{5} B^{k} x-\gamma^{5} B^{k+2} x^{9}, \\
& h(n)=-\gamma^{-5} B^{2-k} n+\gamma^{-5} B^{-k} n^{9}
\end{aligned}
$$

(iv)

$$
\begin{aligned}
& f(x)=\gamma^{5} B^{k} x+\gamma^{5} B^{k+2} x^{9} \\
& g(y)=-B^{2} y+B^{2} y^{3}-B^{2} y^{9}-B^{2} y^{27} \quad \text { and } \\
& f(x)=\gamma^{5} B^{k} x-\gamma^{5} B^{k+2} x^{9}, \\
& h(n)=-\gamma^{-5} B^{2-k} n-\gamma^{-5} B^{-k} n^{9} \\
& h(n)=-\gamma^{-5} B^{2-k} n+\gamma^{-5} B^{-k} n^{9} \text {. }
\end{aligned}
$$

FORM 3. If the subcase (b) of Case 1 occurs (see page 269), proceeding as in the subcase (a), we get autotopisms $(f, g, h)$ where, for $0 \leq k, r \leq 7$,

(i)

$$
\begin{aligned}
& f(x)=B^{k} x^{3}+B^{k+1} x^{27} \\
& g(y)=-\left(\gamma^{5} B^{k+1}+\gamma^{5} B^{r+2}\right) y+\gamma^{5} B^{k} y^{3}+\left(\gamma^{5} B^{k}+\gamma^{5} B^{r+3}\right) y^{9}+\gamma^{5} B^{r} y^{27}, \\
& h(n)=\gamma^{5} n^{3}+\gamma^{5} B^{r-k-1} n^{27} .
\end{aligned}
$$

(ii)

$$
\begin{aligned}
& f(x)=B^{k} x^{3}-B^{k+1} x^{27}, \\
& g(y)=\left(\gamma^{5} B^{k+1}+\gamma^{5} B^{r+2}\right) y+\gamma^{5} B^{k} y^{3}-\left(\gamma^{5} B^{k}+\gamma^{5} B^{r+3}\right) y^{9}+\gamma^{5} B^{r} y^{27}, \\
& h(n)=\gamma^{5} n^{3}-\gamma^{5} B^{r-k-1} n^{27} .
\end{aligned}
$$

(iii)

$$
\begin{aligned}
& f(x)=B^{k} x^{3}+B^{k+3} x^{27}, \\
& g(y)=-\left(\gamma^{5} B^{k+3}+\gamma^{5} B^{r}\right) y+\gamma^{5} B^{k} y^{3}+\left(\gamma^{5} B^{k+2}+\gamma^{5} B^{r+1}\right) y^{9}+\gamma^{5} B^{r} y^{27}, \\
& h(n)=\gamma^{5} n^{3}+\gamma^{5} B^{r-k-3} n^{27} .
\end{aligned}
$$


(iv)

$$
\begin{aligned}
& f(x)=B^{k} x^{3}-B^{k+3} x^{27}, \\
& g(y)=\left(\gamma^{5} B^{k+3}+\gamma^{5} B^{r}\right) y+\gamma^{5} B^{k} y^{3}-\left(\gamma^{5} B^{k+2}+\gamma^{5} B^{r+1}\right) y^{9}+\gamma^{5} B^{r} y^{27}, \\
& h(n)=\gamma^{5} n^{3}-\gamma^{5} B^{r-k-3} n^{27} .
\end{aligned}
$$

CASE 2: One coefficient of $f(x)$ is nonzero. In this case, we get autotopisms $(f, g, h)$ where, for $0 \leq k \leq 7$,

(i)

$$
\begin{aligned}
& f(x)=B^{k} x \\
& f(x)=B^{k} x \\
& f(x)=B^{k} x \\
& f(x)=B^{k} x, \\
& g(y)=y \\
& g(y)=-y \\
& g(y)=B^{2} y \\
& g(y)=-B^{2} y, \\
& h(n)=B^{-k} n \\
& h(n)=-B^{-k} n \\
& h(n)=B^{2-k} n \\
& h(n)=-B^{2-k} n \text {. }
\end{aligned}
$$

(ii)

$$
\begin{aligned}
& f(x)=\gamma^{5} B^{k} x \quad f(x)=\gamma^{5} B^{k} x \\
& f(x)=\gamma^{5} B^{k} x \\
& f(x)=\gamma^{5} B^{k} x, \\
& g(y)=y \\
& g(y)=-y \\
& g(y)=B^{2} y \\
& g(y)=-B^{2} y, \\
& h(n)=\gamma^{-5} B^{-k} n \\
& h(n)=-\gamma^{-5} B^{-k} n \\
& h(n)=\gamma^{-5} B^{2-k} n \\
& h(n)=-\gamma^{-5} B^{2-k} n .
\end{aligned}
$$

(iii)

$$
\begin{aligned}
& f(x)=B^{k} x^{9} \\
& g(y)=y^{9} \\
& h(n)=B^{-k} n^{9}
\end{aligned}
$$$$
f(x)=B^{k} x^{9}
$$$$
g(y)=-y^{9}
$$$$
f(x)=B^{k} x^{9}
$$$$
f(x)=B^{k} x^{9},
$$$$
h(n)=-B^{-k} n^{9}
$$$$
g(y)=B^{2} y^{9}
$$$$
g(y)=-B^{2} y^{9},
$$$$
h(n)=B^{2-k} n^{9}
$$$$
h(n)=-B^{2-k} n^{9} .
$$

(iv)

$$
\begin{array}{llll}
f(x)=\gamma^{5} B^{k} x^{9} & f(x)=\gamma^{5} B^{k} x^{9} & f(x)=\gamma^{5} B^{k} x^{9} & f(x)=\gamma^{5} B^{k} x^{9}, \\
g(y)=y^{9} & g(y)=-y^{9} & g(y)=B^{2} y^{9} & g(y)=-B^{2} y^{9}, \\
h(n)=\gamma^{-5} B^{-k} n^{9} & h(n)=-\gamma^{-5} B^{-k} n^{9} & h(n)=\gamma^{-5} B^{2-k} n^{9} & h(n)=-\gamma^{-5} B^{2-k} n^{9} .
\end{array}
$$

Therefore, there exist only 512 autotopisms from $P$ to $P$ and so the order of $\mathcal{A}(P)$ is 512 . Moreover, 5 is the only 3-primitive prime divisor of $3^{4}-1$ and no autotopism in $\mathcal{A}(P)$ has order 5 .

Finally, suppose that $\mathcal{A}(P)$ is isomorphic to some subgroup $N$ of $\Gamma L(K) \times \Gamma L(K)$. Let's denote $\mathcal{A}_{f}(P), \mathcal{A}_{g}(P)$ and $\mathcal{A}_{h}(P)$ the groups containing the first, second and third components of $\mathcal{A}(P)$. Let's denote $N_{F}$ and $N_{G}$ the groups containing the first and second components of $N$. Then $N_{F}$ and $N_{G}$ are subgroups de $\Gamma L(K)$, and the linear parts of $N_{F}$ and $N_{G}$ are normal subgroups in $\Gamma L(K)$ (see Result 1.21 in [3]), then the linear parts of $\mathcal{A}_{f}(P), \mathcal{A}_{g}(P)$ and $\mathcal{A}_{h}(P)$ are normal subgroups as well, which is a contradiction (see autotopisms in the Case 2, item (i) and item (ii)). Hence, $\mathcal{A}(P)$ is not isomorphic to any subgroup of $\Gamma L(K) \times \Gamma L(K)$.

4. Conclusions. Since the order of the autotopism group of the Figueroa's presemifield of order 81 give in the Theorem 3.1 is 512, we conclude that it is isomorphic to the autotopism group of the semifield plane $\mathbf{P}(\Sigma)$, where $\Sigma$ belong to the Knuth class VIII (see [2]).

\section{ORCID and License}

Walter Meléndez F. http://orcid.org/0000-0002-6253-0205

Moisés Delgado O. http://orcid.org/0000-0002-5267-8169

This work is licensed under the Creative Commons Attribution-NoComercial-ShareAlike 4.0.

\section{References}

[1] Cordero M, Figueroa R. Towards a characterization of the generalized twisted field planes. J. Geom. 1995; 52(1-2):54-63.

[2] Dempwolff U. Semifields Planes of Order 81. J. Geom. 2008; 89(1-2):1-16.

[3] Hughes D, Piper F. Projective Planes. New York: Springer-Verlag; 1973.

[4] Meléndez W, Figueroa R, Delgado M. On the autotopism group of the Cordero-Figueroa semifield of order $3^{6}$. Discuss. Math. - General Algebra and Appl. 2016; 36(1):117-126. doi:10.7151/dmgaa.1250.

[5] Meléndez W, Delgado M. On a conjecture about the autotopism group of the Figueroa's presemifield of order $p^{n}$. Note Mat. 2018; 38(2):11-20. doi: 10.1285/i15900932v38n2p11 